\title{
First Scientific Application of the Membrane Cryostat Technology
}

\author{
David Montanari ${ }^{\mathrm{a}}$, Mark Adamowski ${ }^{\mathrm{a}}$, Bruce R. Baller ${ }^{\mathrm{a}}$, Robert K. Barger ${ }^{\mathrm{a}}$, \\ Edward C. Chi ${ }^{\mathrm{a}}$, Ronald P. Davis ${ }^{\mathrm{a}}$, Bryan D. Johnson ${ }^{\mathrm{a}}$, Bob M. Kubinski ${ }^{\mathrm{a}}$, Ryan \\ Mahoney $^{\mathrm{b}}$, Elaine G. McCluskey ${ }^{\mathrm{c}}$, John J. Najdzion ${ }^{\mathrm{a}}$, Barry L. Norris ${ }^{\mathrm{b}}$, Russel A. \\ Rucinski $^{\mathrm{a}}$, Rich L. Schmitt ${ }^{\mathrm{a}}$, James Stewart ${ }^{\mathrm{d}}$, Terry E. Tope ${ }^{\mathrm{a}}$, Daniel J. Watkins ${ }^{\mathrm{b}}$ \\ ${ }^{a}$ Particle Physics Division, Fermilab, P.O. Box 500, Batavia, IL 60510 USA \\ ${ }^{b}$ Technical Division, Fermilab, P.O. Box 500, Batavia, IL 60510 USA \\ ${ }^{c}$ LBNE Project, Fermilab, P.O. Box 500, Batavia, IL 60510 USA \\ ${ }^{d}$ Physics Department, Brookhaven National Laboratory, P.O. Box 5000, Uptown, NY 11973 USA
}

\begin{abstract}
We report on the design, fabrication, performance and commissioning of the first membrane cryostat to be used for scientific application. The Long Baseline Neutrino Experiment (LBNE) has designed and fabricated a membrane cryostat prototype in collaboration with IHI Corporation (IHI). Original goals of the prototype are: to demonstrate the membrane cryostat technology in terms of thermal performance, feasibility for liquid argon, and leak tightness; to demonstrate that we can remove all the impurities from the vessel and achieve the purity requirements in a membrane cryostat without evacuation and using only a controlled gaseous argon purge; to demonstrate that we can achieve and maintain the purity requirements of the liquid argon during filling, purification, and maintenance mode using mole sieve and copper filters from the Liquid Argon Purity Demonstrator (LAPD) R\&D project. The purity requirements of a large liquid argon detector such as LBNE are contaminants below 200 parts per trillion oxygen equivalent. This paper gives the requirements, design, construction, and performance of the LBNE membrane cryostat prototype, with experience and results important to the development of the LBNE detector.
\end{abstract}

\section{INTRODUCTION}

The Long Baseline Neutrino Experiment (LBNE) project envisions using membrane tank technology for a large liquid argon detector referred to as the Far Detector (FD) with the start of the construction in the time frame of 2020. Membrane tank technology is significantly more cost effective than flat plate vessel construction for a cryogenic tank of this volume $\left(\sim 7,000 \mathrm{~m}^{3}\right)$ [1]. The LBNE cryostat in its current configuration is $28.56 \mathrm{~m}$ long, $15.63 \mathrm{~m}$ wide and $15.98 \mathrm{~m}$ high (inner dimensions). It will be instrumented with Time Projection Chambers (TPCs) and filled with liquid argon.

In order for the TPCs to operate properly and drift electrons with a lifetime greater than $1.4 \mathrm{~ms}$, the LBNE-FD requires extreme purity of the liquid argon inside the tank, at the level of 200 parts per trillion (ppt) of oxygen equivalent contamination. The empirical correlation between electron lifetime and parts of oxygen equivalent contamination is outlined in equation 1 [2]. Cryogenic vessels for particle physics research are typically pumped down to remove impurities prior to filling. However, it is highly impractical to pump down a tank of the size of the LBNE-FD. An R\&D project called Liquid Argon Purity Demonstrator (LAPD) proved that a slow gaseous argon purge can remove impurities down to parts per million from a large cylindrical vessel without evacuation. With a liquid argon filtration system composed by a mole sieve and copper filters, it reached over $5.0 \mathrm{~ms}$ electron lifetime [2], far exceeding the level of purity for the liquid argon required by the LBNE-FD.

$$
\text { Lifetime }[\mathrm{ms}]=\frac{3 \cdot 10^{-13}[\mathrm{~ms} \cdot \text { parts of Oxygen }]}{\text { Contaminant }[\text { parts of Oxygen }]}
$$

We have built a small prototype $\left(\sim 29 \mathrm{~m}^{3}\right)$ to demonstrate the possibility of fabricating a membrane cryostat that meets the requirements for a low background particle physics detector: thermal performance, feasibility for liquid argon, argon purity, and leak tightness. We also wanted to check the feasibility of construction interfaces and 
methods, including the business aspects of negotiating a contract with a large membrane cryostat supplier. The membrane cryostat manufacturer is required to have a safe, reliable design with a proven track record. History shows that membrane cryostats are the technology of choice for Liquefied Natural Gas (LNG) transportation and storage, with hundreds of ship tankers and storage tanks built and being built all over the world with sizes up to $250,000 \mathrm{~m}^{3}$ (Fig. 1 [3]), much larger than the LBNE-FD. It is a commercially available technology and has been in service for several decades without a single recorded incident. This prototype, known as the LBNE 35 ton prototype, was competitively procured for the design, purchase of materials and supervision during the installation. IHI Corporation (IHI) won the bid.

\section{DESCRIPTION}

The LBNE 35 ton prototype is the first and only membrane cryostat built for scientific purposes, and the first and only membrane cryostat built in the United States. While membrane cryostat technology is widely used for LNG, this prototype will also be the first liquid argon. The temperatures of liquid argon and LNG are very similar, with the latter less than $30 \mathrm{~K}$ warmer than the former, but the densities are quite different: LNG is about one third less dense. LNG ship tankers and storage tanks are more than four times taller than LBNE-FD (and more than twenty times taller than this prototype), making the two applications very similar. The hydrostatic pressure at the bottom of the tank is almost identical.

A membrane cryostat is made of several parts (Fig. 2): the corrugated membrane that contains the liquid and gaseous Argon, the passive insulation that reduces the heat leak, and the reinforced concrete structure that is the structural part to which the pressure is transferred. A secondary barrier system embedded in the insulation protects it from potential spills of liquid argon, and a vapor barrier over the concrete protects the insulation from the moisture of the concrete (Fig. 3a) $[4,5]$. A roof made by two plates, A and B, completes the cryostat. Plate A is a flat plate with insulation and membrane underneath. Plate $\mathrm{B}$ is a flat plate containing all the penetrations and services.

The membrane cryostat has been designed by IHI and constructed by Fermilab personnel under IHI supervision. The concrete structure has been designed by an independent contractor following Fermilab and IHI specifications and constructed by a civil construction company. The mechanics of the top plate have been designed by Fermilab in consultation with IHI and fabricated by Fermilab personnel.

The LBNE 35 ton prototype shares a liquid Argon filtration system with the LAPD project.

The basic idea was to simulate as many features as possible of the future LBNE-FD. We chose a square tank with 5\% ullage and a volume comparable to LAPD. The cryostat is 4.0 meters long, 2.7 meters wide, and 2.7 meters high (inner dimensions). The design parameters outlined in Table 1 are the same as those for LBNE-FD with the exception of the insulation thickness, the type of vapor barrier, and the duration of the project. For example, due to building constraints, it was neither practical nor necessary to build this tank with 0.8 meter of insulation, so we chose 0.4 meter, which corresponds to double the thickness of a standard insulation panel. The size of LBNE-FD will make the use of a metallic vapor barrier very time consuming and expensive, so we chose to use epoxy or resin. The anticipated duration of LBNE-FD is 20 years, not 10 years as is the case for this project.

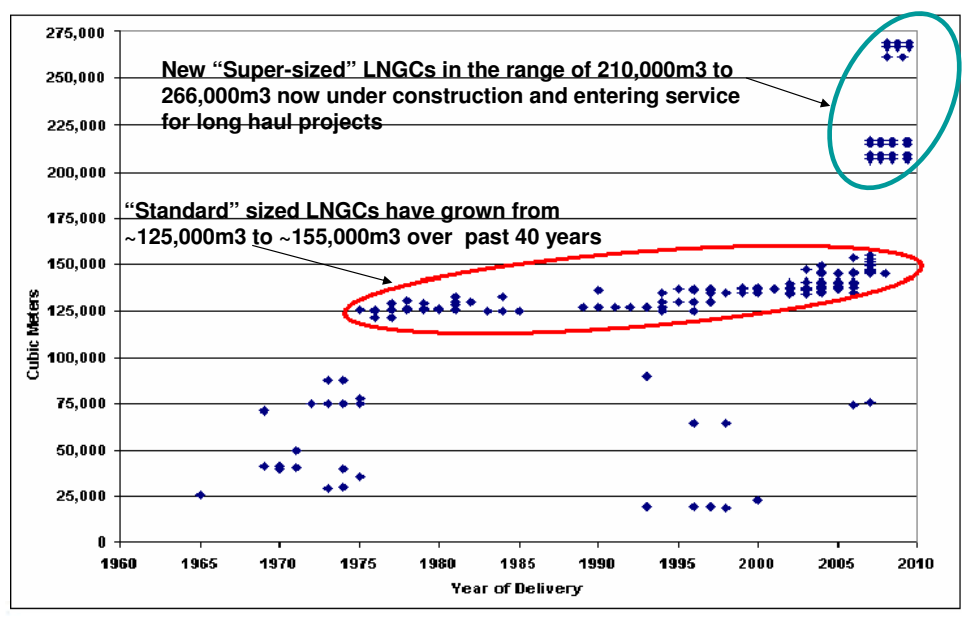

FIGURE 1. Capacity of LNG tanks in the past 50 years. Reprinted from Peter G. Noble, A short history of LNG shipping 19592009, Texas Section, SNAME, 2009. 


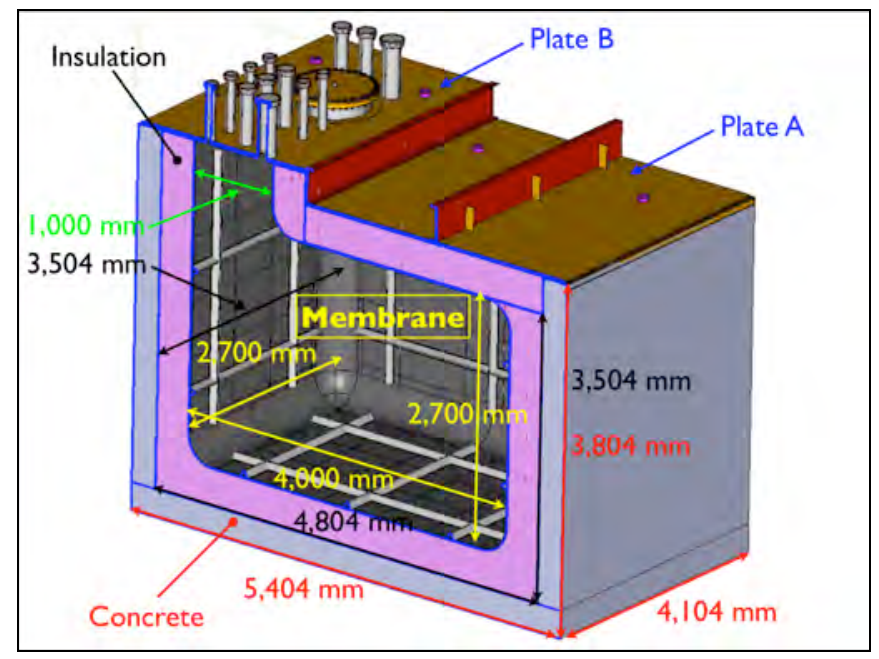

FIGURE 2. Membrane cryostat 3D drawing.

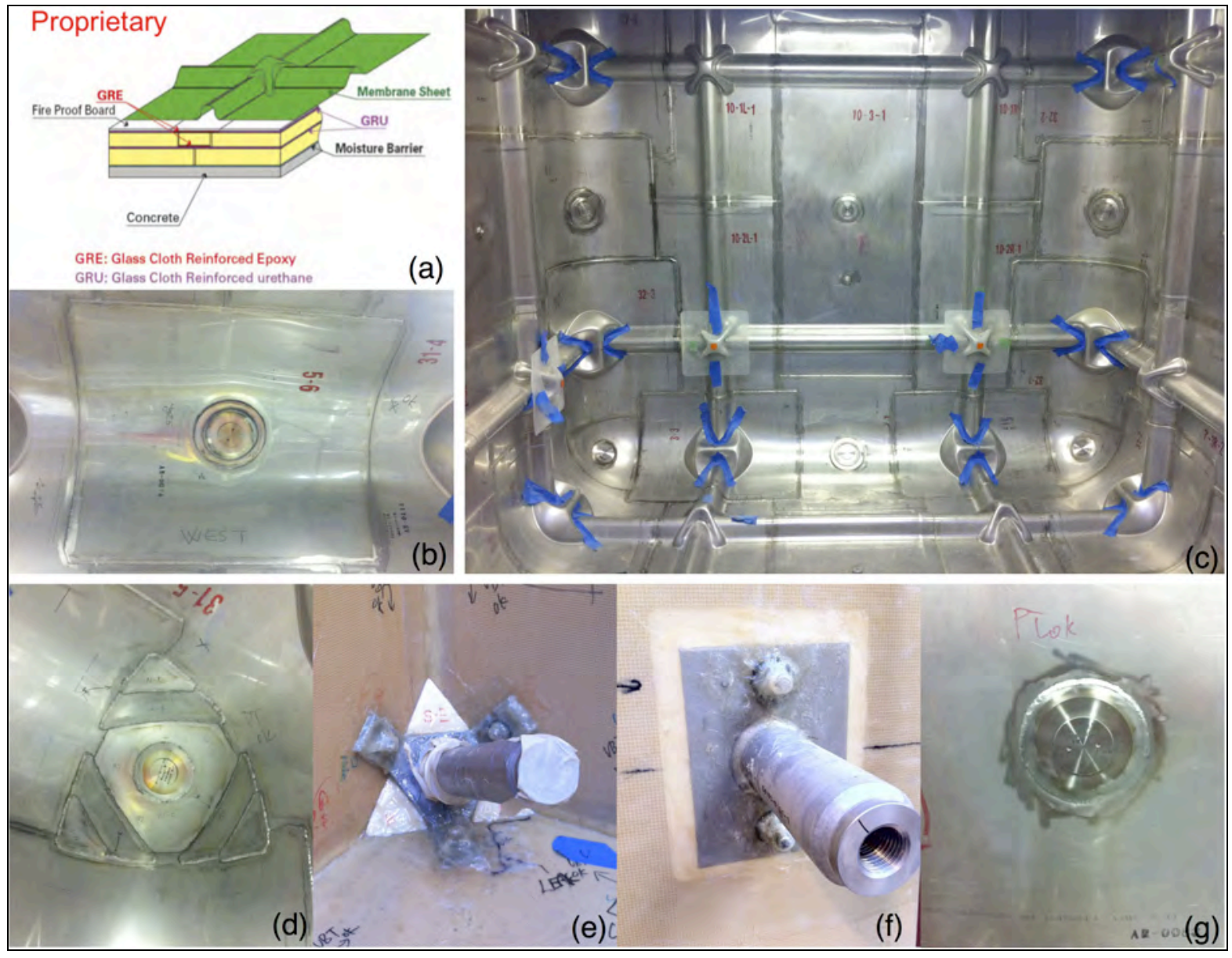

FIGURE 3. Membrane cryostat construction photos: (a) Membrane cryostat section, reprinted from IHI Corporation [5]; (b) Membrane panel in angle with screw; (c) Inside of the tank after completion; (d) Membrane panel in corner with screw; (e) Three-way anchor; (f) One-way anchor, and (g) Flat membrane panel with screw. 
TABLE 1. Design parameters for the LBNE 35 ton prototype.

\begin{tabular}{lc}
\hline Design Parameter & Value \\
\hline Cryostat volume & $29.16 \mathrm{~m}^{3}$ \\
Liquid argon total mass & $38,600 \mathrm{~kg}$ \\
Inner dimensions of the cryostat & $2.0 \mathrm{~m}(\mathrm{~L}) \times 2.7 \mathrm{~m}(\mathrm{~W}) \times 2.7 \mathrm{~m}(\mathrm{H})$ \\
Depth of liquid argon & $2.565 \mathrm{~m}(5 \%$ ullage $)$ \\
Insulation & $0.4 \mathrm{~m}$ Polyurethane foam \\
Primary membrane & $0.1 \mathrm{~mm}$ thick fiberglass \\
Secondary barrier system & $1.2 \mathrm{~mm}$ thick carbon steel \\
Vapor barrier & $0.3 \mathrm{~m}$ thick \\
Reinforced outer concrete layer & $89 \mathrm{~K}+/-1 \mathrm{~K}$ \\
Liquid argon temperature & $70 \mathrm{mBar}(\sim 1 \mathrm{psig})$ \\
Operating gas pressure & No vacuum \\
Vacuum & $207 \mathrm{mBar}(\sim 3 \mathrm{psig})$ \\
Design pressure & $1 \mathrm{E}-06 \mathrm{mBar} 1 / \mathrm{sec}$ \\
Leak tightness & $<13 \mathrm{~W} / \mathrm{m}^{2}$ \\
Heat leak & 10 years \\
Duration & thick SS 304 corrugated stainless steel \\
Thermal cycles & Fermilab ES\&H \\
Design codes & Acomplicable parts of JGA RP-107-02 \\
& ACI 318 \\
\hline
\end{tabular}

\section{Concrete}

A reinforced concrete outer shell ensures the structural resistance to the liquid argon load, gas pressure and external loads, such as the weight of the top plate and of all the instruments and services connected to it. These loads are transferred through the insulation from the membrane to the concrete structure. The bottom slab has been equipped with heaters and temperature controllers to maintain the concrete temperature within acceptable limits. The sidewalls and the bottom have been poured in place and are 0.3 meter thick reinforced with rebar. On the inside there is a vapor barrier made by $1.2 \mathrm{~mm}$ thick carbon steel sheets welded to angles embedded in the concrete. The angles on the sides and the bottom are steel, and the angles at the top of the sides are stainless steel to guarantee the required leak tightness, the cleanliness and the cryogenic service (Fig. 4a). Stainless steel cover plates $(9.5 \mathrm{~mm}$ thick) have been welded to the angles over the sides of the concrete structure. The vapor barrier has been leak checked with a helium mass spectrometer in sniffer mode and any leak repaired. Helium has been injected in the region between the concrete and the metal sheets through temporary holes. Prior to helium leak checking, the vapor barrier was tested with vacuum boxes: snoop solution is poured over the weld seam and partial vacuum is pulled inside a box placed over the region of interest; if bubbles come out of the welding seam, there is a leak. Any identified leak was repaired (Fig. 4b).

Several stainless steel rods pass through the vapor barrier and are anchored to the concrete. They hold the insulation and the membrane anchors and are welded to carbon steel sheets.

\section{Insulation}

The insulation thickness is driven by the required heat leak. We have chosen a heat leak lower than $15 \mathrm{~W} / \mathrm{m}^{2}$ : that value can be achieved with $0.4 \mathrm{~m}$ of standard polyurethane foam insulation. In reality, there are two layers of insulation: a layer outside ( $0.2 \mathrm{~m}$ thick) and a layer inside $(0.19 \mathrm{~m}$ thick). The actual calculated heat flux value is $11.5 \mathrm{~W} / \mathrm{m}^{2}$, without the contribution of plate $\mathrm{B}$, which includes the penetrations. The standard insulation panels are 1 meter wide, 2 meters long and are joined together with liquid polyurethane foam that cures and hardens forming a single continuous layer.

A secondary barrier system is embedded over the outer layer of insulation: it covers the bottom and sides up to the liquid level height inside the tank in order to contain the liquid argon in case of a primary membrane failure. A sub-secondary barrier system is embedded over the inner layer of insulation, with the same layout and purpose. 
Secondary and sub-secondary barrier systems are made by Glass Reinforced Urethane (GRU) and are bridged together with Glass Reinforced Epoxy (GRE) to form two continuous layers.

The insulation panels are bolted to the concrete through stainless steel rods and nuts that are also covered with liquid insulation and GRE.

A $10.0 \mathrm{~mm}$ thick calcium silicate fireproof board glued over the sub-secondary barrier system protects the insulation from the high heat generated during the welding (Fig. 4c and Fig. 4d).

Secondary and sub-secondary barrier systems have been tested with vacuum boxes: snoop solution is poured over the joint seam (between two adjacent panels or around a rod) and partial vacuum is pulled inside a box placed over the region of interest; if bubbles come out of the seam, there is a leak. Any identified leak has been repaired.

The insulation is normally purged with gaseous argon to ensure that, in case of a leak in the membrane, air will not enter the system. A distribution system is located between the insulation and the membrane, under the corrugations. It has two inlets, two outlets, and radial holes in the pipes.
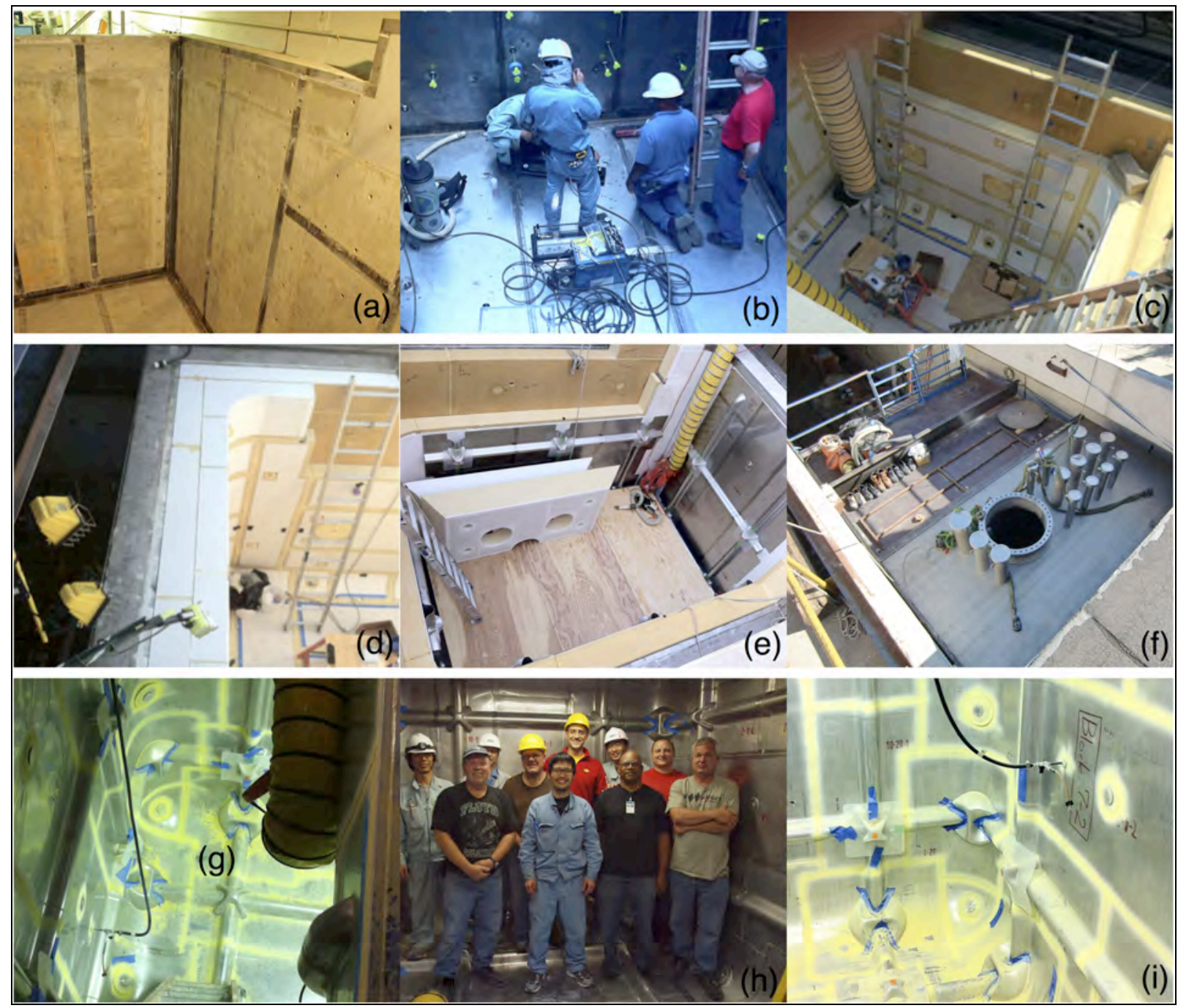

FIGURE 4. Photos during construction: (a) Concrete structure before starting the installation of the vapor barrier; (b) Test of the vapor barrier with vacuum box; (c) Installation of the inner layer of insulation; (d) Top view during the installation of the second layer of insulation; (e) Installation of the membrane; (f) Top view of the completed membrane cryostat; (g) Ammonia leak check of the membrane cryostat; (h) Partial group photo after the completion of the installation, and (i) Ammonia leak check of the membrane cryostat. 


\section{Membrane}

The membrane panels are $2.0 \mathrm{~mm}$ thick 304 corrugated stainless steel. They are fixed to the concrete with screws specifically designed to engage membrane anchors. Longitudinal and latitudinal corrugations absorb the thermal contraction and expansions of the metal with temperature.

The flat member panels were pre-welded at the vendor's site in five wall size sections for the sides and floor (Fig. 4e). They were then "TIG" welded with double passes to membrane panels for corners and angles.

The membrane anchors are bolted to membrane anchor rods over the outer layer of insulation (Fig. 3e). The inner layer of insulation has matching holes to accept the anchors and they are then filled with liquid insulation. The membrane is then screwed into the membrane anchors (Fig. 3b, Fig. 3d, Fig. 3g). Three types of membrane anchors were used: one-way (Fig. 3f) for flat membrane panels; two-way, for angle panels; and three-way for corner panels (Fig. 3e). The screws going into the two-way and three-way anchors need a backing washer to adapt to the uneven surface of the membrane panel. The head of the screws were also welded to the membrane panels.

\section{Roof}

Two flat plates constitute the roof of the LBNE 35 ton prototype: plate A and plate B. Plate A is a $19.0 \mathrm{~mm}$ thick carbon steel plate reinforced with two C-channels and equipped with insulation and the membrane. It was assembled upside down, and then flipped and lowered on top of the cryostat. The insulation and membrane were installed following the same process as for the sides and bottom with the anchor rods for the insulation welded to the top plate. Six anchors are welded to the plate; they pass through the insulation and terminate with a welded connection to the membrane. TPCs will be hung from the anchors during a second phase of the project. Plate B is a $25.4 \mathrm{~mm}$ thick 304 stainless steel plate with no insulation and membrane. It sits on a higher portion of the cryostat $(0.3 \mathrm{~m}$ taller than the rest) and contains all the penetrations and services that are connected to the tank, which include liquid and gaseous argon inlets and outlets, a pressure relief valve, temperature and purity monitors, condenser, and a manhole to access the tank after completion. Radiation shields reduce the heat leak underneath plate B. Plates A and $\mathrm{B}$ are welded to the cover plates that sit on top of the concrete. Plate $\mathrm{B}$ is removable by design to allow the insertion of TPCs into the cryostat and attach them under plate A later in the project.

\section{Membrane leak check}

At the end of the installation, the membrane was tested with dye penetrant (for superficial cracks) and with a colorimetric leak check (for actual leaks); both ASTM standard Non Destructive Testing (NDT) methods. The colorimetric leak check has a measured sensitivity of $1 \mathrm{E}-06 \mathrm{mBar} * 1 / \mathrm{sec}$. A mixture of ammonia and nitrogen was injected in the insulation region in closed loop, through special holes drilled in the membrane panels that were welded after the completion of the test. The return path was the gaseous argon purge piping, and a pump continuously circulated the gas mixture. A paint developer that changes color from yellow to blue if in contact with Ammonia in concentration greater than $3 \%$, is sprayed over the weld seams inside the tank to detect potential leaks and their location. We periodically measured and recorded the concentration of ammonia behind every wall (sides, roof and floor). Two control holes painted with the developer were also connected to the wall with the lowest concentration of ammonia. The paint turned blue in the 10 micron hole and remained yellow in the 5 micron hole, as expected. No leak was recorded and the membrane cryostat was approved for pressure testing (at $259 \mathrm{mBarg}$ or $125 \%$ of design pressure), which was successfully performed.

\section{CRYOGENIC SYSTEM}

The LBNE 35 ton prototype cryogenic system is composed of several parts: a liquid argon purification system, a gaseous argon filtration system, an argon condenser with liquid nitrogen cooling system, and a liquid and gaseous argon supply system (Fig. 5).

We will reuse the existing LAPD cryogenic system to purify the liquid argon, composed of mole sieves and copper bed filters, to remove water and oxygen respectively. While the LAPD tank and the LBNE 35 ton prototype vessel cannot operate at the same time, it will be possible to transfer the liquid from one to the other. We will also reuse the liquid and gaseous argon supply system, and the gaseous argon filtration system [2]. 
The membrane cryostat will have its own condenser to re-condense the boil off argon from the tank. It will be a duplicate of LAPD, but located on top of the membrane cryostat. Since it has been demonstrated that all the impurities are in the ullage at the top of the tank $[2,4,6]$, the re-condensed liquid will then go to the purification system before entering the tank.

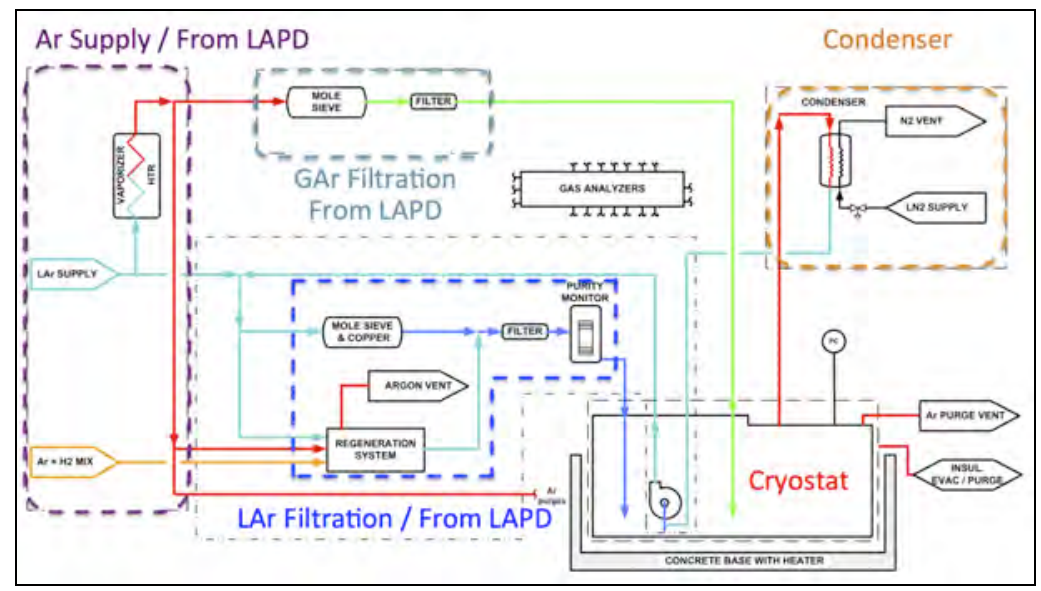

FIGURE 5. Process flow diagram for the LBNE 35 ton prototype cryogenic system

The main difference between LAPD and the LBNE 35 ton cryogenic system is the use of liquid argon submerged pumps located inside the membrane cryostat and hung from plate B. They are a commercial product, typically used in LNG applications.

The piping to connect the membrane cryostat to the existing filters is being fabricated. Once the construction is completed, the cryostat will be slowly purged with gaseous argon to reduce the impurities (we are mostly concerned with oxygen and water) to parts per millions. Then the liquid filling and circulation begins and will reduce the impurities to the required parts per trillions of oxygen equivalent contamination, or more than $1.4 \mathrm{~ms}$ electron lifetime.

\section{CURRENT STATUS}

The LBNE 35 ton prototype membrane cryostat has been constructed and successfully pressure tested. The cryogenic system that connects the tank to the LAPD purification system is being fabricated with a target completion in the summer of 2013, the commissioning and testing program will then begin. The LBNE 35 ton prototype is the test facility for the LBNE project, and the only prototype that will be built before the actual detector. There is an extensive test program that comprises two phases. Phase 1 main goal is to demonstrate the purity of the liquid argon, while Phase 2 is to build a prototype of the detector.

More specifically, the goals of Phase 1 are:

- To demonstrate the membrane cryostat technology, in terms of thermal performance, feasibility for liquid argon, leak tightness.

- To demonstrate that we can achieve and maintain the purity requirements in a membrane cryostat without evacuation, less than $200 \mathrm{ppt}$ oxygen equivalent contamination, or more than $1.4 \mathrm{~ms}$ electron lifetime

- $\quad$ To identify design needs and changes to LBNE-FD.

Also, the goals of Phase 2 are:

- $\quad$ To insert TPCs inside the tank and take data, such as purity and electronic noise.

- To insert cables and test possible contamination to the purity of the liquid argon.

We have already accomplished the first goal of checking the feasibility of construction interfaces and methods, and the business aspects of negotiating a contract with a large membrane cryostat supplier. 


\section{FUTURE ACTIVITIES}

The LBNE 35 ton prototype is the first and only membrane cryostat built for scientific purposes as well as the only membrane cryostat built in the United States. High energy physics and low background experiments are building larger and larger detectors and are potentially interested in this technology: membrane cryostat technology is cost effective when the linear dimension is equal to or exceeds 10 meters. There are no scheduled activities after the completion of Phase 2, scheduled for completion by the end of 2014. We have envisioned a Phase 3 where the prototype is available to scientists and researchers other than LBNE.

\section{ACKNOWLEDGMENTS}

Operated by Fermi Research Alliance, LLC under Contract No. De-AC02-07CH11359 with the United States Department of Energy.

\section{REFERENCES}

1. Russel A. Rucinski (private communication), 2012.

2. Mark A. et al., "Extreme argon purity in a large non-evacuated cryostat", CEC-ICMC 2013, 2013

3. Peter G. Noble, "A short history of LNG shipping 1959-2009", Texas Section, SNAME, 2009.

4. Ishikawajima-Harima Heavy Industries Co. Ltd, "GBS Membrane”, 2005.

5. IHI Corporation (private communication), 2011.

6. Brian J. Rebel (private communication), 2012. 